\title{
An integrated analysis of microRNAs involved in fat deposition in different pig breeds
}

\author{
Xiuxiu Zhang ${ }^{1}$, Wanlong Huang ${ }^{1}$, Yuntao Guo ${ }^{1}$ and Xiangyang Miao ${ }^{1}$ \\ ${ }^{1}$ State Key Laboratory of Animal Nutrition, Institute of Animal Sciences, Chinese Academy of Agricultural Sciences, Beijing, \\ 100193, China
}

Correspondence to: Xiangyang Miao, email: miaoxy32@163.com, mxy32@sohu.com

Keywords: pig; RNA-seq; miRNA; adipose tissue; PPAR

Received: January 04, $2017 \quad$ Accepted: November 01, $2017 \quad$ Published: December 01, 2017

Copyright: Zhang et al. This is an open-access article distributed under the terms of the Creative Commons Attribution License 3.0 (CC BY 3.0), which permits unrestricted use, distribution, and reproduction in any medium, provided the original author and source are credited.

\begin{abstract}
The domestic pig, an important species in the animal production industry, is also a model system for studying fat deposition and fat-associated diseases in humans. In order to investigate the genetic relationships of the miRNAs that may regulate fat deposition, we performed a genome-wide analysis of miRNAs derived from subcutaneous adipose tissue of Laiwu and Large White pig using RNA-seq. A total of 26,486,906 reads were obtained. Further analysis indicated that 39 known miRNAs and 56 novel miRNAs had significantly differential expression between the two breeds of pigs. Gene Ontology and KEGG pathway analysis revealed that the predicted targets of these differentially expressed miRNAs were involved in several fat-associated pathways, such as the PPAR, MAPK and Wnt signaling pathways. In addition, we found that three miRNAs, sSc-miR-133a-3p, ssc-miR-486 and ssc-miR-1, had an impact on the development of porcine subcutaneous fat through the PPAR signaling pathway. This study provides clues to understand the potential mechanisms of adipogenesis and fat deposition between two different pig breeds. Furthermore, these results may also contribute to the research involving human obesity.
\end{abstract}

\section{INTRODUCTION}

Obesity is a heterogeneous disease that is associated with comorbidities, such as type 2 diabetes mellitus (T2DM), cardiovascular disease and cancer [1]. It has been a major risk for human health. Therefore, it is urgent to elucidate the mechanism of obesity and develop effective treatment programs. In general, adipose tissue (AT) is considered a passive reservoir for energy storage that also functions in mechanical and heat insulation or participates in the regulation of thermogenesis. However, recently, it is recognized that AT synthesizes and secretes a variety of bioactive peptides, known as "adipokines," which participate in the regulation of glucose and lipid metabolism, energy homeostasis, feeding behavior, insulin sensitivity, inflammation, immunity, adipogenesis and vascular function or coagulation [2]. Several studies have examined the role of dysfunctional adipose tissue in the pathogenesis of obesity. Therefore, the study of adipose tissue function is of great significance for revealing the mechanism of obesity.
The realization of adipose tissue function is closely related to adipocyte differentiation and fat metabolism. Previous studies showed that adipose cell differentiation and fat metabolism were regulated strictly by several transcription factors (TFs), including sterol regulatory element binding proteins (SREBPS), CCAAT/enhancer binding proteins (C/EBPS) and peroxisome proliferatoractivated receptor gamma (PPAR $\gamma)$. Recent studies indicated that microRNAs (miRNAs) also play critical roles in the adipose cell differentiation and fat metabolism. miRNAs are a set of small noncoding RNAs (1822nt) that bind to partially complementary sequences on target mRNAs resulting in the posttranscriptional regulation of gene expression [3]. They play vital roles in cell differentiation, apoptosis, metabolism and other important biological processes [4-7]. And these include their effects on adipocyte differentiation and fat deposition. For example, previous observations [6] argue that miR-14 is a dose-dependent regulator of DAG and TAG metabolism in Drosophila. Esau et al. reported miR-143 is involved in adipocyte differentiation [8]. In 
addition, bta-miR-378 promotes the differentiation of bovine preadipocytes [9], ssc-miR-7134-3p regulates fat accumulation in castrated male pigs [10], miR-429 inhibits differentiation in porcine preadipocyte and so on [11]. In this study, we identified the miRNAs which affect the adipocyte differentiation and fat metabolism in pigs.

The pig is an excellent model for studying obesity and diseases associated with obesity. One of the reasons for this is that pigs have the ability to generate fatty deposits in a manner that is similar to humans. Moreover, the development, morphology and function of the normal cardiovascular system in swine closely resemble that of humans [12]. Pigs are also, like humans, omnivores, and develop spontaneous atherosclerosis with increased age and have lipoprotein profiles and a metabolism that is similar to humans [13]. The Laiwu pig, a typical obese pig and that easily deposits fat, is a unique breed in the Shandong Province of China with characteristics of high adaptability, meat quality, fecundity and a strong combining ability. Large White pigs, also called Yorkshire pigs, originate from the United Kingdom and have the characteristics of a large size, high fecundity and adaptability, and high feed conversion rate and dressing percentage. Large White pigs are one of the world-class lean meat-type pigs. These two breeds show significant differences in fat accumulation and serve as ideal animal models for studying fat deposition and obesity.

Recently, there has been an increase in the utilization of Next Generation Sequencing (NGS) to examine the transcriptome for the identification of differential expression as well as for the opportunity to discover novel transcripts, including new alternative isoforms and miRNAs [14-26]. Therefore, in the present study, a comparison of the miRNA profiles of the adipose tissue of the Laiwu pig and Large White pig was performed using RNA-seq. The data were further analyzed to identify the miRNAs that were related to adipopexis. This study provides information on the miRNAs that are involved in the accumulation of fat in pigs, and the target genes and pathways they regulate. Swine producers use genetics to rear pigs that have rapid lean growth. However, a balance has to be maintained as breeding pigs with a high proportion of lean meat has led to a decrease in the intramuscular fat content and therefore decreased pork quality. This study sheds light on the players in the adipose pathway that could be manipulated to rear pigs which show rapid lean growth accompanied by optimum intramuscular fat. Moreover, as the genetics of fat deposition is similar between pigs and humans, the data obtained in this study would also provide clues for solving the problems related to human obesity.

\section{RESULTS}

\section{miRNAs sequencing and mapping}

We performed the miRNA sequencing on an Illumina Hiseq 2500 platform, and the reads were generated from the L library and D library. A total of approximately 26,486,906 raw reads were obtained. After removing the low-quality reads, adaptors and all possible contaminations, we obtained a total of 16,813,692 uniquely mapped reads, among which 8,259,953(64.09\%) were from the L library and 8,553,739 (62.89\%) were from the D library. The clean reads were mapped to the Rfam database and were distributed among several main categories, including miRNAs, rRNAs, snRNAs, tRNAs, other small RNAs and unannotated small RNAs (Table 1). The reads mapped to the unannotated small RNAs made up the highest proportion. The reads mapped to the miRNAs were the second most common $(12.43 \%$ in L library and $14.28 \%$ in D library). The reason for the majority of the reads mapped to unannotated small RNAs may be due to the fact that these are not well studied and annotated.

\section{Identification of known miRNAs and novel miRNAs}

After mapping to the Rfam database, the remaining sequences were used for annotation of the conserved and novel miRNAs. In the Laiwu pig, 2660 known miRNAs and 584 novel miRNAs were identified. In the Large White pig, 2634 known miRNAs and 561 novel miRNAs were identified. Among them, 1851 miRNAs were common in both breeds. The novel miRNAs in this study were named by the following rules: species name-m (four-digit)-5p/3p, for example, ssc-m0644-3p. A statistical analysis of the sequence length of the mature miRNAs indicated that the lengths were mainly distributed at about $22 \mathrm{nt}$, which is a typical size range for Dicer-derived products and is in line with the principle of miRNA processing.

\section{Identification of differentially expressed miRNAs}

The Laiwu pig and the Large White pig showed distinct fat depositions. To investigate the miRNAs that may be responsible for the significant differences in the fat characteristics, we identified the differentially expressed miRNAs between the Laiwu pig and Large White pig. The strict screening criteria are mentioned in the method section. The results indicated that 17 known miRNAs, including ssc-miR-133a-3p, ssc-miR-486, ssc-miR-1 and ssc-miR-204, were up-regulated in the Laiwu pig compared to the Large White pig (Supplementary Table 1). Meanwhile, 22 known miRNAs, including ssc-let-7i and ssc-miR-27b-3p, were down-regulated in the Laiwu pig compared to the Large White pig (Supplementary Table 2). In addition, ssc-miR-133a-3p, ssc-miR-486, ssc-miR-1, ssc-let-7i and ssc-miR-27b-3 were abundantly expressed in the Laiwu pig compared to the Large White pig. In addition, we identified 55 differentially expressed novel miRNAs between the Laiwu pig and the Large White pig. These differentially expressed miRNAs were used for further prediction of the targets. 
Table 1: Statistics of the classification of the small RNAs

\begin{tabular}{ccc}
\hline Type & D libraries & L libraries \\
\hline Unannotation & $6,463,227$ & $6,054,417$ \\
miRNA & $1,221,683$ & $1,026,545$ \\
rRNA & 229,610 & 234,485 \\
snRNA & 189,944 & 156,546 \\
tRNA & 222,328 & 564,823 \\
other & 226,947 & 223,137 \\
\hline
\end{tabular}

\section{Target gene prediction of the differentially expressed miRNAs}

The identification of miRNA targets helps to illustrate the functions of the differentially expressed miRNAs identified in the present study. A total of 36,259 genes were predicted to be the potential targets of the 94 differentially expressed miRNAs in the Laiwu and Large White pig (Supplementary Table 3 and Supplementary Table 4). miRNAs can bind to partially complementary sequences on target mRNAs and result in the posttranscriptional regulation of gene expression, thus changing the biological processes in which their target genes are involved. Therefore, in order to further explore the function of the differentially expressed miRNAs, we mainly utilised the targeted genes for the subsequent functional analysis.

\section{Gene ontology (GO) and KEGG pathway analysis of the targeted genes of differentially expressed miRNAs}

GO terms were assigned to the differentially expressed miRNAs targeted genes based on their sequence similarities to known proteins in the UniProt database annotated with GO terms as well as the InterPro and Pfam domains they contain. The GO annotation and enrichment analysis of these targeted genes were done using the DAVID software (Supplementary Table 5 and Supplementary Table 6), in which the gene length bias was corrected. The GO terms with corrected $P$-value less than 0.05 were considered significantly enriched by the targeted genes. The distribution bar charts of the biological processes, cellular components and molecular functions are shown in Figure 1. From the perspective of the biological processes, the targeted genes of the up-regulated differentially expressed miRNAs were significantly enriched in cellular protein complex assembly (GO: 0043623) and protein complex biogenesis (GO: 007027). The targeted genes of the down-regulated differentially expressed miRNAs were significantly enriched in gluconeogenesis (GO: 000609), hexose biosynthetic processes (GO: 0019319) and carbohydrate biosynthetic process (GO: 0034637) and other GO terms related with material synthesis and energy metabolism. From the molecular function perspective, the targeted genes of the up-regulated and down-regulated differentially expressed miRNAs were mainly enriched in steroid binding (GO: 0005496), lipid binding (GO: 0008289) and protein phosphatase activity, which are related to the functions of enzyme activity and lipid binding. From the cellular component perspective, cytoskeleton (GO: 0005856) and organelle (GO: 0043228) were significantly enriched by the targeted genes of both the up-regulated and downregulated differentially expressed miRNAs.

To further probe the potential function of the miRNAs with differential expression, we used the DAVID software to test the statistical enrichment of the targeted genes of the differentially expressed miRNAs in the Kyoto Encyclopedia of Genes and Genomes (KEGG) pathway (Figure 2 and Supplementary Table 7). Figure 2 shows the results of the pathway enrichment, which clearly displays that the MAPK, PPAR and Wnt signaling pathways are the significantly enriched terms. This suggests that by targeting the genes in these pathways known to be involved in fatty acid metabolism, the differentially expressed miRNAs might regulate adipogenic differentiation and lipid metabolism.

\section{Construction of the miRNA target network}

To better display the interaction between the miRNAs and their target genes, we selected the target genes enriched in both significant GO BP terms and KEGG pathways and the genes reported to be associated with fat deposition to construct the miRNA target networks [27-29]. As is shown in Figure 3 and Supplementary Table 8 , one gene can be targeted by multiple miRNAs, and most miRNAs target more than one target gene. For example, ssc-miR-455-3p was directed to the targeted genes CPT1A and DKK3 and ssc-miR-874 was directed to STARD3 and MGLL. Importantly, among these targeted genes, SCD, SCD5, STARD3 and CPT1A are reported to be involved in adipocyte differentiation and fat deposition [27-29].

\section{The relationship between differentially expressed miRNAs and the targeted genes in the PPAR pathway}

As mentioned above, three important factors in the PPAR signaling pathway, SCD, SCD5 and CPT1A, 
participate in adipocyte differentiation and fat deposition and also are predicted to be the targets of ssc-miR-1, sscmiR-486 and ssc-miR-133a-3p, respectively (Figure 4A). In addition, the expressions of these three miRNAs were highly abundant. Therefore, we described the relationships between the three differentially expressed miRNAs and the targeted genes in the PPAR pathway based on the STRING database. Figure $4 \mathrm{~B}$ shows the complicated relationships between glycerol kinase (GK), peroxisome proliferator-activated receptor delta (PPARD), diazepam binding inhibitor(DBI), acyl-CoA binding protein, stearoyl-CoA desaturase (SCD), SCD5, cytochrome P450 family 7 subfamily A, polypeptide 1(CYP7A1), solute carrier family 27 member 2 (SLC27A2) and Carnitine palmitoyltransferase 1A (CPT1A) in the PPAR pathway. The miRNAs were also included in the analysis in order to illustrate the potential regulators. Therefore, as Figure 4B displays, ssc-miR-133a-3p, ssc-miR-1 and ssc-miR-486 might change the signal transduction in the PPAR signaling pathway by targeting the important genes CPT1A, SCD and SCD5, respectively, and thus, take part in the regulation of biological processes related to adipogenesis.

\section{Transcription factor binding sites (TFBS) prediction}

The predicted results showed that the promoter regions of the precursors of ssc-miR-486, ssc-miR-133a-3p and sscmiR-1 (Table 2) had multiple transcription factor binding sites, including Jun proto-oncogene (JUN), transcription factor AP-2 (AP-2), Sp1 transcription factor (SP1), Sp2 transcription factor (SP2), sterol regulatory element binding transcription factor 1 (SREBF1,SREBP1), sterol regulatory
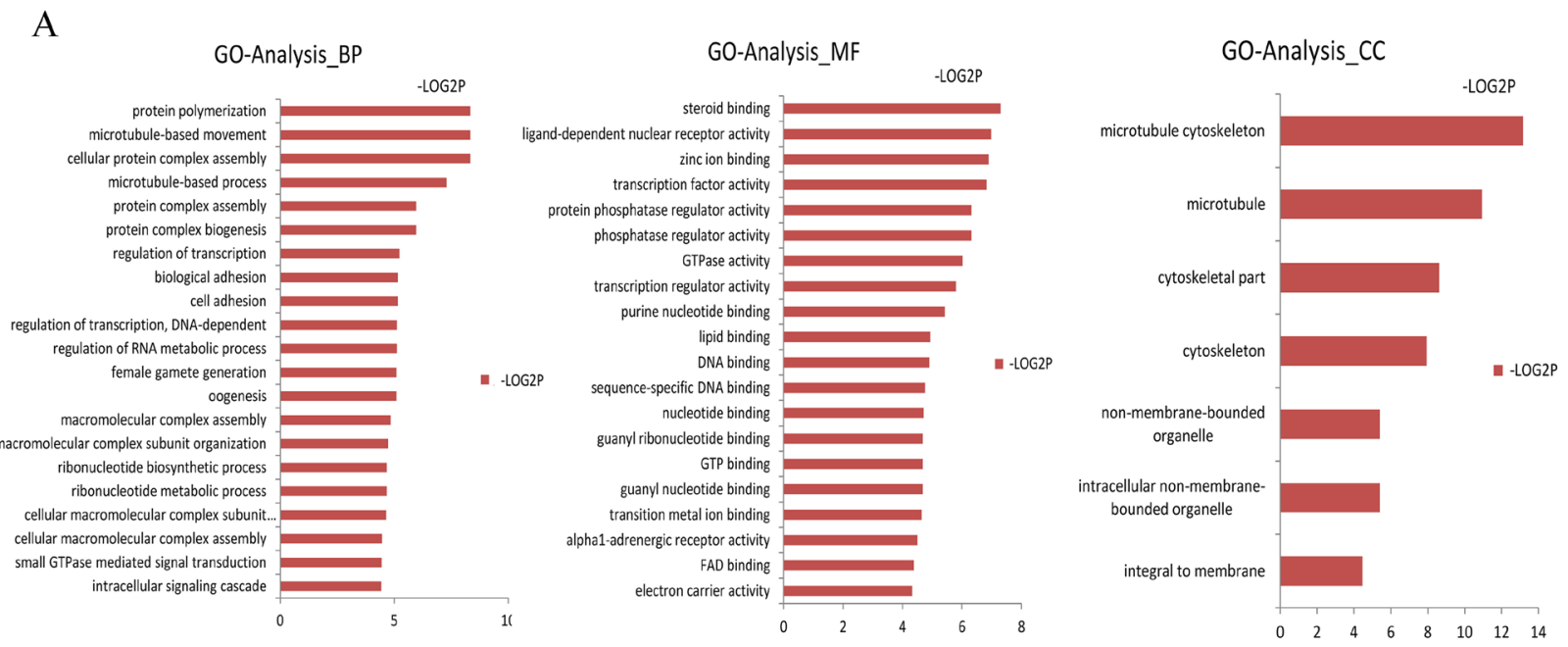

B
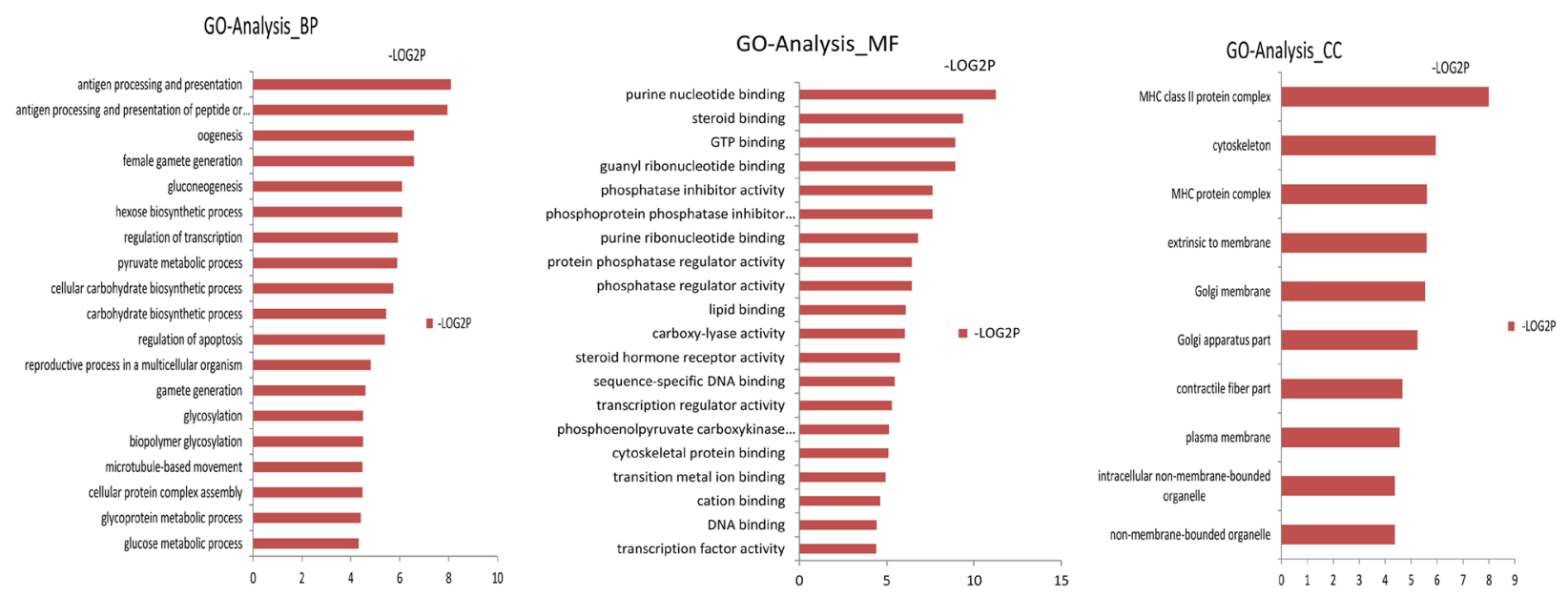

Figure 1: GO analysis of the targeted genes of the differentially expressed miRNAs. (A) represents the GO analysis of the targeted genes of the up-regulated differentially expressed miRNAs. (B) represents the GO analysis of the targeted genes of the downregulated differentially expressed miRNAs. The figure is composed of three parts: biological processes (BP), molecular functions (MF), and cellular components (CC). The significance level of enrichment was set at a corrected $p<0.05$. 
element binding transcription factor 2 (SREBF2,SREBP2), CCAAT/enhancer binding protein alpha (CEBPA), CCAAT/ enhancer binding protein beta (CEBPB), myogenic differentiation 1 (Myod1), SRY-box 17 (Sox17) and runtrelated transcription factor 2 (RunX2) (Figure 4C). Of these transcription factors, SP1, SREBP1, SREBP2, CEBPA and CEBPB are involved in the proliferation and differentiation of preadipocytes and in lipid metabolism. These data suggest that these transcription factors might bind to the promoter regions of ssc-miR-486, ssc-miR-133a-3p and ssc-miR-1 to further regulate the adipose-related biological processes.

\section{Validation of the sequencing data by qRT-PCR}

To validate the sequencing data, six differentially expressed miRNAs were randomly selected and were further examined by qRT-PCR (Supplementary Table 9). The result showed that ssc-miR-486, ssc-miR-133a-3p, ssc-miR-122, ssc-miR-1, ssc-miR-204 and ssc-m0092$3 p$ were significantly higher in the Laiwu pig. These data were consistent with the sequencing results.

\section{DISCUSSION}

Over one-third of the world population is now struggling with overweight or obesity issues, and a disease burden frequently associated with these conditions [30]. Obesity is a well-known risk factor for metabolic diseases, such as T2DM, dyslipidemia, coronary heart disease, hypertension, non-alcoholic fatty liver disease (NAFLD) and stroke. It is also linked to dementia, obstructive sleep apnea and numerous types of cancers [31]. Therefore, revealing the mechanism of fat deposition and adipocyte differentiation is of great significance for the treatment of obesity. Previous studies have focused on the mechanism of fatty deposits using rodents. For example, the rat 3T3L1 cell line is usually used to study the differentiation of fat cells [32, 33]. Moreover, fat mice are also considered as animal models to study fat metabolism diseases [34]. However, there is a gap in the fatty deposit locations and fatty deposits formation between humans and rodents. Pigs, with a maximum ability for fat deposition, are the most obese animals and are similar to humans in their manner of fat deposition. Therefore, pigs are ideal animal models to study human diseases related to adipose [13]. Recently, researchers have studied the mechanism of obesity using pigs and made some progress [35]. In this study, the Laiwu pig and Large White pig, with significant differences in adipopexis, were chosen as the animal models. The profiles of the miRNAs in the adipose tissue of these two different breeds were generated using RNAseq to further study the potential molecular mechanism of adipopexis. The results indicated that 40 miRNAs were up-regulated in the Laiwu pig, and meanwhile, 54 miRNAs were down-regulated in the Laiwu pig. The bioinformatics analysis of these differentially expressed miRNAs provided more information about the identification, classification and the possible targeted genes that might be related to adipopexis.

Previous studies have shown that some differentially and abundantly expressed miRNAs (down-regulated miRNAs: ssc-let-7i, ssc-miR-21, ssc-miR-27b-3p and up-regulated miRNAs: ssc-miR-1, ssc-miR-133a-3p, ssc-miR-486) were responsible for regulating adipogenic differentiation [36-38, 41-43]. ssc-miR-1 and ssc-miR$133 \mathrm{a}$ were found extensively in skeletal muscle tissues while ssc-miR-21, ssc-miR-27b-3p, and the Let-7 family play important roles in adipomuscle and myocardial tissue. Evidence suggests that these miRNAs are found in muscular tissues [39, 40]. However, in this study, ssc-miR-1 and ssc-miR-133a, with highly abundant expression, were up-regulated in the adipose tissue of the Laiwu pig. Moreover, previous studies suggest that ssc-miR-1 and ssc-miR-133a are related to adipocyte differentiation in humans and rats [41, 42]. Therefore, ssc-miR-1 and ssc-miR-133a may increase lipometabolism in pigs. In addition, the concentration of miR-486 in the serum is correlated with T2DM and obesity, and miR-486, as a circulating miRNA, may be related with metabolic disorders [43]. Thus, the up-regulation of ssc-miR-486 in Laiwu pigs may be a potential biomarker of obesity.

Some differentially expressed miRNAs with a low expression were also related with adipopexis, including ssc-miR-758, ssc-miR-144, ssc-miR-204 and ssc-miR-122. ssc-miR-122 plays an important role in regulating cholesterol and maintaining the steady state of fatty acids. The depressed expression of miR-122 effectively reduces the cholesterol content in the serum [44]. Furthermore, the targeted control of ssc-miR-144 and ssc-miR-758 to the ATP-binding cassette sub-family A member 1 (ABCA1) gene results in an indirect regulation of the metabolism of cholesterol [45, 46]. ssc-miR-204 inhibits the Runx2 gene, thus, accelerating the adipogenic differentiation of MPCs and BMSCs [47], and the upregulation of ssc-miR-204, in this study, indicates that it may activate adipocyte differentiation in pigs. In addition, for the first time, we found that ssc-miR-206, ssc-miR331-3p, ssc-miR-451, ssc-miR-499-5p, ssc-miR-885 and ssc-miR-95 were up-regulated in Laiwu pigs, and sscmiR-218b, ssc-miR-411, ssc-miR-450c-5p, ssc-miR-452, ssc-miR-455-3p, ssc-miR-542-3p, ssc-miR-136 and sscmiR-1285 were down-regulated in the Laiwu pigs. These miRNAs are more extensively studied in cancer, but are seldom looked at in lipometabolism [48-51].

The GO enrichment analysis suggested that the targeted genes of the differentially expressed miRNAs were enriched in carbohydrate biosynthesis, lipid biosynthesis, steroid binding, enzyme activity and other functions involved in lipometabolism and energy metabolism. The KEGG enrichment analysis indicated that the PPAR, MAPK, Wnt and TGF-beta signaling pathways were enriched. Previous studies showed 
Table 2: The precursor information of ssc-miR-486, ssc-miR-133a-3p and ssc-miR-1

\begin{tabular}{ccccc}
\hline Name & Accession & Chromosome & Location & Length(bp) \\
\hline ssc-mir-486-1 & NC_010459 & 17 & $12191266-12191345$ & 80 \\
ssc-mir-486-2 & ENSSSCG00000022807 & 17 & $12191229-12191310$ & 82 \\
ssc-mir-133a-1 & ENSSSCG00000018790 & 17 & $69274653-69274755$ & 103 \\
ssc-mir-133a-2 & ENSSSCG00000026111 & 6 & $99485202-99485288$ & 87 \\
ssc-mir-1 & ENSSSCG00000019004 & 17 & $69285393-69285500$ & 108 \\
\hline
\end{tabular}

that these signaling pathways are related to adipocyte differentiation and fat metabolism. MAPK signaling pathway involves four pathways, including ERK1/2, JNK, p38 MAPK and ERK5/BMK1, which have played an important role in adipopexis [52-55]. The Wnt signaling pathway is involved in the fate determination of the fat cell, adipogenic differentiation and lipometabolism [56, 57]. Kennsell et al. argued that the Wnt signaling pathway inhibited adipogenic differentiation either by depending on beta-catenin or not [58]. The TGF-beta signaling pathway is also closely related with lipometabolism. Kim et al. suggested that miR-21 targeted the gene transforming growth factor beta receptor 2 (TGFBR2) to regulate adipogenic differentiation in human stem cells
[36]. Moreover, we noticed that PPAR signaling pathway is important in fatty acid metabolism, sterol metabolism and adipogenic differentiation. Figure 4 shows that CPT1A, SCD5 and SCD (SCD1) are enriched in this pathway, and they are targeted by ssc-miR-133a-3p, sscmiR-486 and ssc-miR-1, respectively. SCD, with highly conserved sequences, exists in two forms of isomers, SCD1 and SCD5, in most vertebrates [59]. SCD1 is the limiting enzyme in the transformation of saturated fatty acids to monounsaturated fatty acids, which plays an important role in fatty acid biosynthesis $[14,60]$. SCD5 affects the components of aliphatic acid in the milk of Holstein cows, which is also an important regulatory gene in lipometabolism [61]. CPT1 has three different

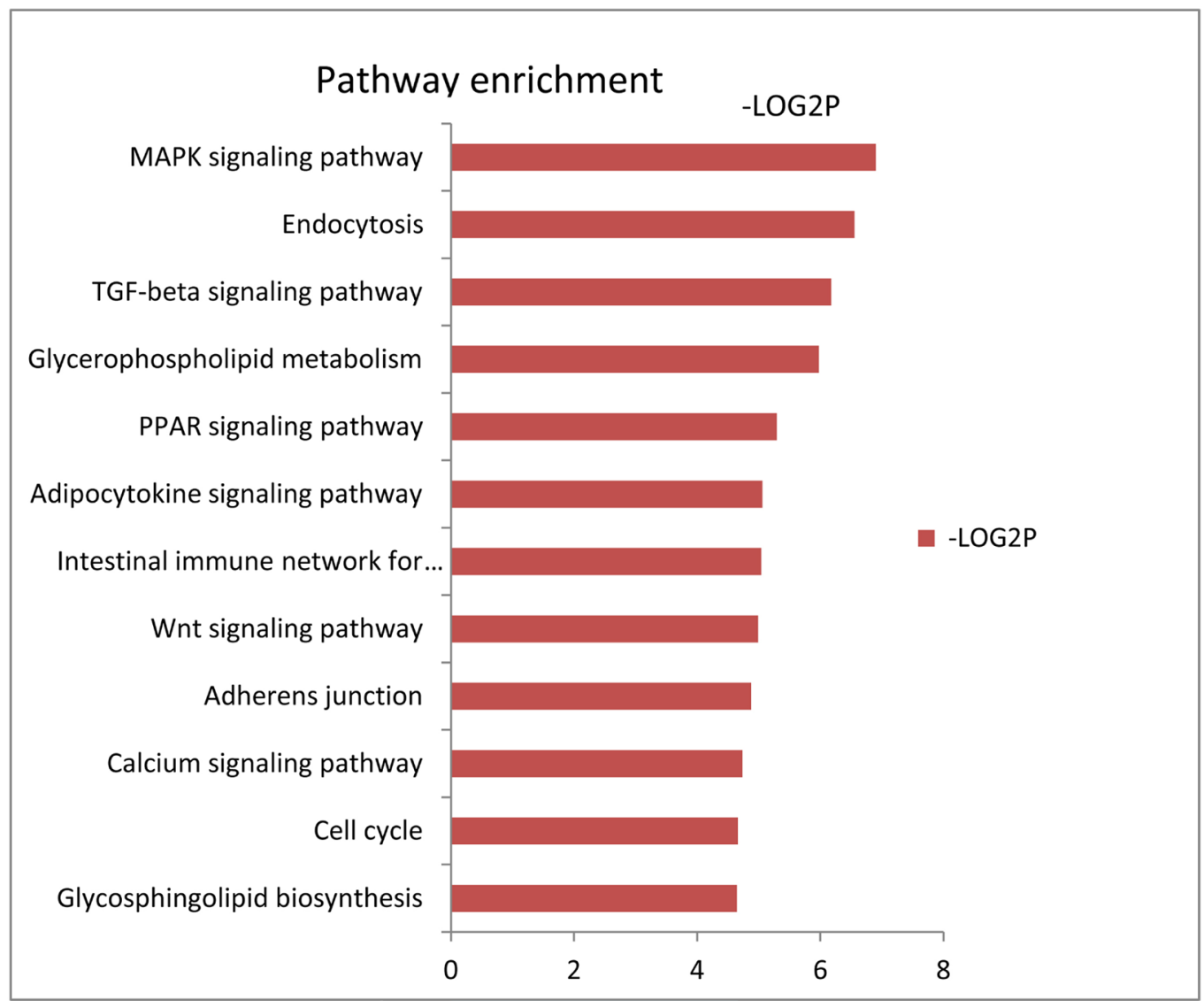

Figure 2: KEGG enrichment analysis of the targeted genes of the differentially expressed miRNAs. The horizontal axis refers to the $-\log 2 \mathrm{P}$, while the vertical axis refers to the significantly enriched pathway with a corrected $p<0.05$. A higher number for $\log 2 P$ value indicates the promoted performance of the pathway enrichment. 
hypotypes, including CPT-1A, CPT-1B and CPT-1C. CPT1 is the limiting enzyme in the beta-oxidation of free fatty acids. CPT1 catalyzes long-chain fatty acyl coenzyme A and carnitine into acyl carnitine, which facilitates in the oxidation of fatty acids in mitochondria matrix [29]. Malandrino et al. found that CPT1A promotes the oxidation of fatty acids in fat cells and macrophages, thus, reducing lipid accumulation and inflammation [62]. Therefore, CPT1A plays a role in the inhibition of fat deposition. Based on the evidence mentioned above, sscmiR-133a-3p, ssc-miR-486 and ssc-miR-1 may regulate lipid metabolism by targeting the genes CPT1A, SCD5 and SCD, respectively. Among these, ssc-miR-133a-3p may promote the fat deposition in pigs by targeting the gene CPT1A, which is in line with the high expression and up-regulation of ssc-miR-133a-3p in Laiwu pigs. However, how ssc-miR-486 and ssc-miR-1 effect lipid metabolism and deposition in the pig by targeting SCD5 and SCD requires further investigation. To validate the RNA-seq data, we selected six differentially expressed miRNAs and determined the expression of these RNAs by RT-PCR (Supplementary Table 9). We used three animals in each of the two breeds for the miRNA validation. Each miRNA's expression/fold changes for the Large White and Laiwu pig groups were compared. The expression levels obtained by RT-PCR were consistent with the RNA-seq results described above. Taken together, these findings suggest that the identified miRNA-targeted genes contribute to regulating genes and pathways that are involved in adipogenesis, which may help to define the fat deposition differences between two groups of pigs.

Recently, it has been shown that targeting PPARs is a potential way to treat NAFLDs $[63,64]$. Since the miRNAs that we uncovered in our study regulate the PPAR pathway, this study helps us understand the players involved in the regulation of this pathway and could provide insights into better treatment for NAFLDs.

\section{MATERIALS AND METHODS}

\section{Ethics statement}

All of the procedures involving animals were approved by the animal care and use committee at Institute of Animal Sciences, Chinese Academy of Agricultural Sciences where the experiment was conducted. All of the experiments were performed in accordance with the relevant guidelines and regulations set by the Ministry of Agriculture of the People's Republic of China.

\section{Sample preparation}

All the animals in this study were maintained in Laiwu Pig Farm (Shandong, China), and two different breeds were analyzed: Laiwu pig and Large White pig. They were housed under the same conditions, including free access to water and food in natural lighting. Three healthy pigs from each breed (150-day-old) were selected for the study. At slaughter age, the mean body weights of Laiwu pigs and Large White pigs were $35 \mathrm{~kg}$ and $90 \mathrm{~kg}$ respectively. The subcutaneous adipose tissues were collected after the animals were butchered, and these tissues were frozen in liquid nitrogen and stored immediately at $-80^{\circ} \mathrm{C}$ for further RNA extraction. All the experimental procedures were carried out according to authorization granted by the Chinese Ministry of Agriculture.

\section{Library preparation and miRNA sequencing}

Total RNA was extracted from the subcutaneous adipose tissues of two different breeds using TRIzol (Invitrogen) as described by the manufacturer. The RIN or RNA integrity number was used to determine the RNA integrity quality of the extraction. Qualified total RNA was stored at $-80^{\circ} \mathrm{C}$ for later miRNA separation. The deep-sequencing technology was used for the RNASeq [65]. Two small RNA libraries representing the two species (coming from a pool of 3 Laiwu pigs and a pool of 3 Large White pigs) were constructed according to the Illumina ${ }^{\circledR}$ TruSeq $^{\mathrm{TM}}$ Small RNA Sample Preparation protocol. Briefly, small RNAs were isolated from the total RNA by a denaturing polyacrylamide gel electrophoresis (PAGE). The 5'-adaptor and 3'-adaptor were ligated to these small RNAs, followed by reverse transcription with SuperScript II Reverse Transcriptase (Invitrogen), and then, the cDNA was amplified by PCR and was purified to generate two small RNA libraries. Quality control analysis on the library was performed with the Qubit ${ }^{\mathrm{TM}}$ dsDNA HS kit and a Qubit ${ }^{\circledR}$ 2.0 Fluorometer. High Sensitivity DNA Chip and Agilent 2100 system were used to determine the library size and purity. Finally, the qualified small RNA libraries were sequenced using the single-read multiplex program at the Beijing Biotechnology Corporation (Beijing, China). The two cDNA libraries from the two species (coming from a pool of 3 Laiwu pigs and a pool of 3 Large White pigs) were named as $\mathrm{L}$ and $\mathrm{D}$, respectively.

\section{Mapping and annotation}

Reference genome and gene model annotation files were downloaded from the genome website directly. To analyze the miRNA sequencing results, the adaptor sequences and low quality sequences were initially filtered out from the raw reads by the fastx_toolkit (http:// hannonlab.cshl.edu/fastx_toolkit/). The clean reads were first aligned to the pig genome (NCBI, Sscrofa10.2) using the bowtie program (version 1.0.0) and were then searched against the Rfam database (http://rfam.xfam. org/) to remove rRNA, tRNA and snRNA. Moreover, the remaining sequences were compared with the mature miRNAs of human and animals, including cow, sheep, 
and pig in miRBase (Release 21; http://www.mirbase. org/) to identify the conserved miRNAs. The hits were considered a real match if there was a minimum 16-nucleotide match between the sequence read and the miRNA from the database. The read sequences that matched with the reference mature miRNAs (allowing for one or two mismatching end-nucleotides) were annotated as miRNA candidates. The miRNA candidates were then clustered into categories according to sequence similarity, and the sequences varying only in length and/or a few end nucleotides were grouped under the same miRNA identifier or related keywords, which is typically used to identify miRNAs (https://www.mdc-berlin.de/36105849/ en/research/research_teams/systems_biology_of_gene regulatory_elements/projects/miRDeep/documentation).

\section{Identification of novel miRNAs}

To identify potential novel miRNAs in pigs, 150 nucleotides of a sequence flanking each side of these miRNA candidate sequences were extracted. The secondary structures were then predicted using the program MIREAP (http://sourceforge.net/projects/ mireap/). If a hairpin structure with a free energy of hybridization lower than $-20 \mathrm{kcal} / \mathrm{mol}$ was predicted, the RNA sequence was subjected to the miReap analysis, which predicts whether the input RNA sequence is a genuine pre-miRNA-like hairpin sequence. Any sequence that met the following three criteria was harvested: (1) mature miRNAs reside in one arm of the hairpin precursor, which are short of large internal loops or bulges; (2) the stem-loop structure is steady, with the free energy hybridization lower than $-20 \mathrm{kcal} / \mathrm{mol}$; and (3) hairpins located in intergenic regions or introns were considered as potential new miRNAs [66].

\section{Differential expression analysis}

In order to obtain the differentially expressed miRNAs, the RPM method was used to judge the expression level of the two samples. The differential expression analysis of miRNAs was performed using the DEGSeq R package (1.10.1). DEGSeq provided statistical routines for determining the differential expression in the digital gene expression data using a model based on the binomial distribution. The resulting $P$-values were corrected using the Benjamini and Hochberg's approach for controlling the false discovery rate. Differentially expressed miRNAs with a corrected $P$-value $<0.05$ and a more than 2-fold difference were selected for further analysis.

\section{Target prediction of the differentially expressed miRNAs}

In mammals, miRNAs incompletely complementarily pair with the 3 '-UTR region of the target genes to repress the transcription of key regulators
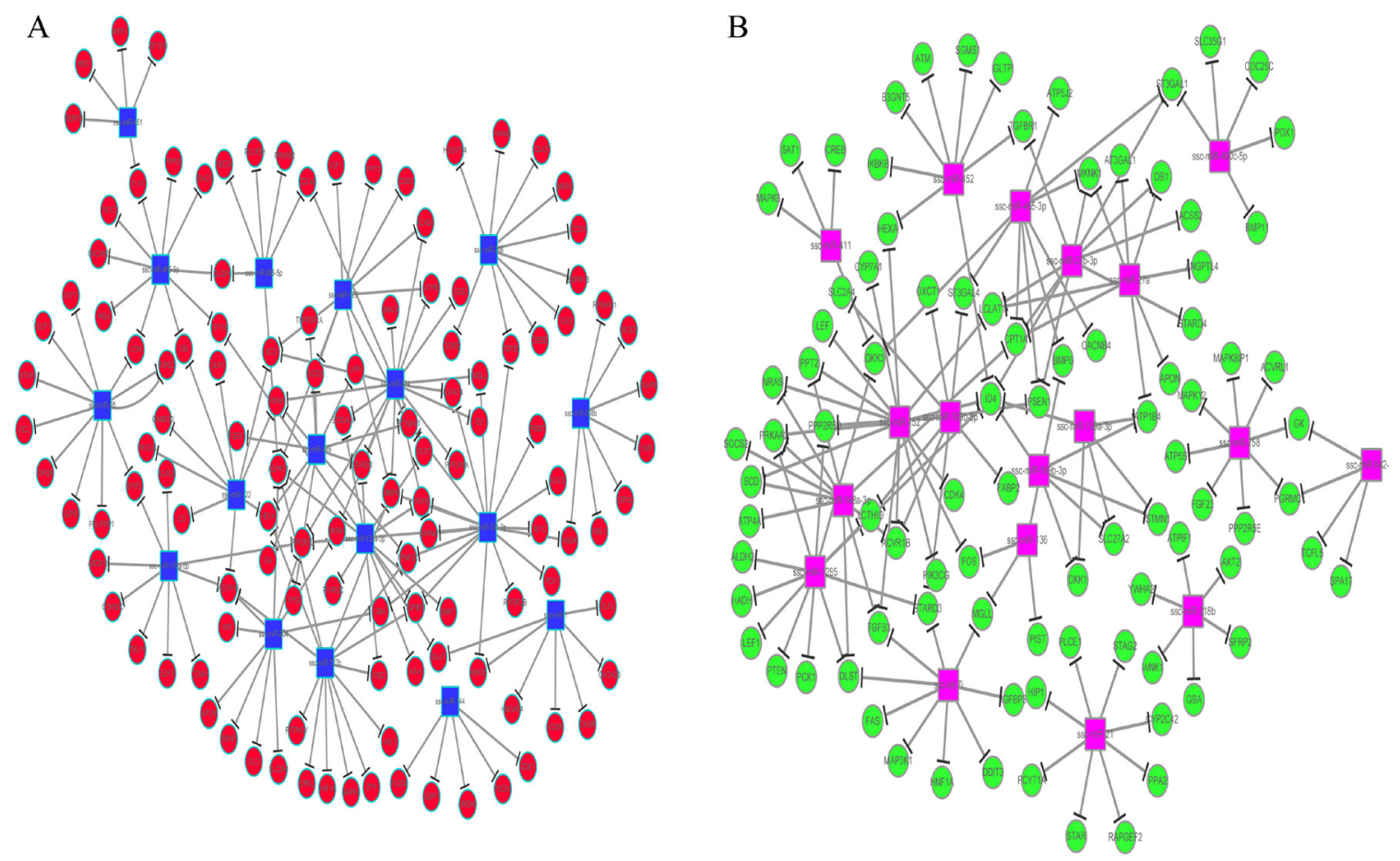

Figure 3: Relationships between the differentially expressed miRNAs and their targeted genes. (A) represents the relationships between the up-regulated miRNAs with their targeted genes. (B) represents the relationships between the down-regulated miRNAs with their targeted genes. The circular nodes represent the targeted genes, while the square nodes represent the differentially expressed miRNAs. 
related to growth, differentiation and other important pathways. The second base to the eighth base of the miRNAs are generally considered the 'seed' sequence, which is important to recognize and bind the 3 '-UTR region of their targets. Therefore, to explore the potential function of the miRNAs with the significantly differential expression in porcine subcutaneous adipose tissue, the miRanda program (http:/www. microrna.org/microrna/ getDownloads.do) was applied to predict their target mRNAs in the linux system based on the complementary pairing of bases. The 3'-UTR sequences were extracted according to gene annotation information. The parameters were set as follows: score threshold at 140; energy threshold at- $19 \mathrm{kcal} / \mathrm{mol}$; scaling parameter at 4 and gapopen penalty at-9. A set of predicted target genes was designed for further analysis.

\section{GO and KEGG pathway analyses of the miRNA target genes}

The GO enrichment analysis of the target genes of the differentially expressed miRNAs was implemented by the DAVID software (https://david.ncifcrf.gov/). With all the porcine genes as the list of the background genes, the GO terms with corrected $P$-value less than
0.05 were considered significantly enriched terms. KEGG is a database resource for understanding highlevel functions and utilities of the biological system, such as the cell, the organism and the ecosystem, from molecular-level information, especially for large-scale molecular datasets generated by genome sequencing and other high-throughput experimental technologies (http:// www.genome.jp/kegg/). We used the DAVID software to test the statistical enrichment of the target genes of the differentially expressed miRNAs in the KEGG pathways. The KEGG terms with corrected $P$-values less than 0.05 were considered significantly enriched terms with the list of background genes of all porcine genes.

\section{Interaction networks of miRNA and the target genes}

Based on the String database (http://string-db.org/), we displayed the interaction networks of the miRNAs and their target genes in the PPAR signaling pathway and illustrated the relationships of the miRNAs with their target genes in this pathway. Moreover, in order to better demonstrate the relationships between differentially expressed miRNAs with their target genes, Cytoscape software was used to assess the relationships between

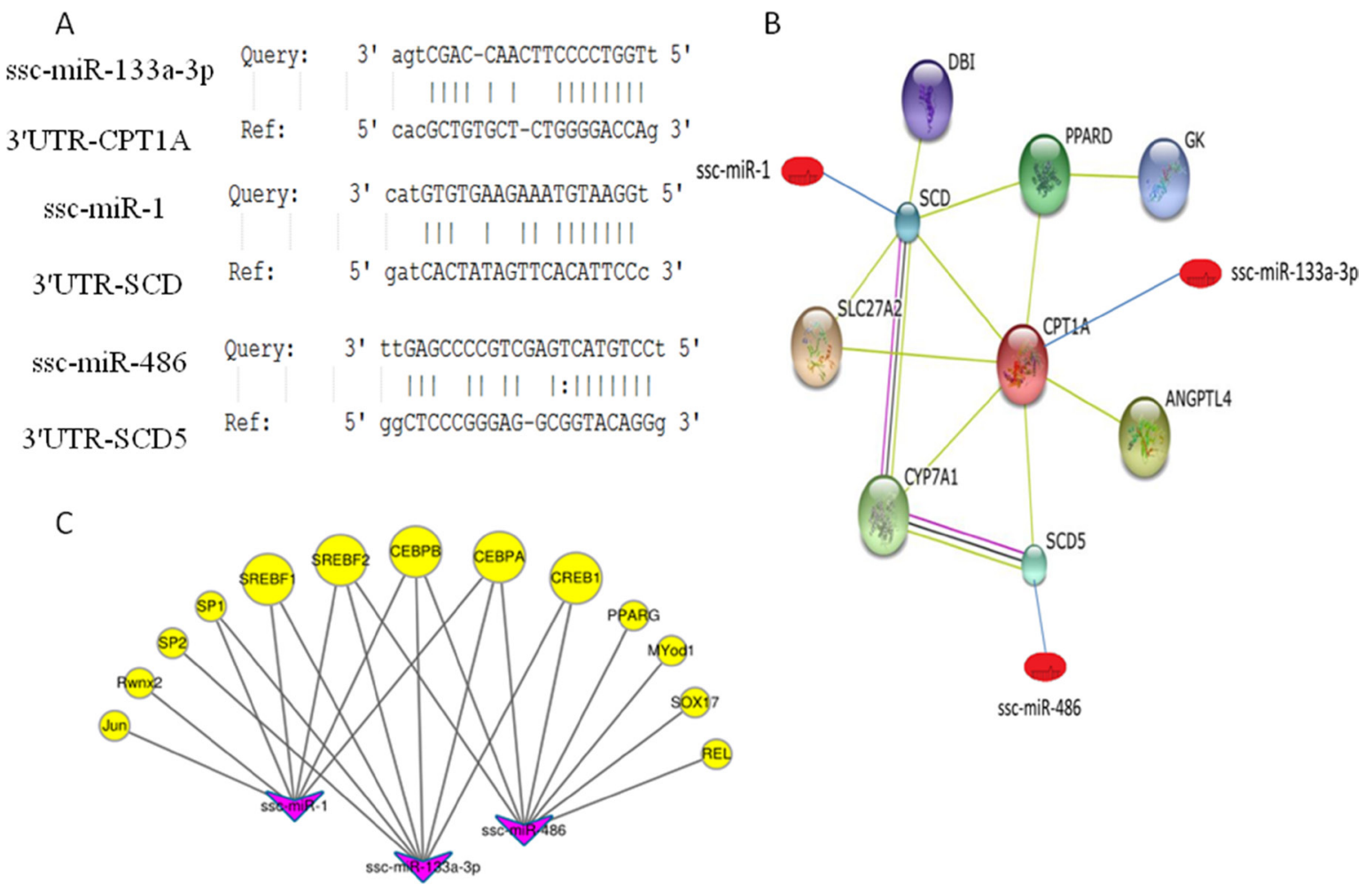

Figure 4: Relationships between ssc-miR-133a-3p, ssc-miR-1 and ssc-miR-486 and the targeted genes CPT1A, SCD and SCD5 and the transcription factors binding to these miRNAs. (A) represents the result of the target gene prediction of sscmiR-133a-3p, ssc-miR-1 and ssc-miR-486 using miRanda software. (B) represents the protein-protein interactions and the interactions of the miRNAs with the CPT1A, SCD and SCD5 genes in the PPAR pathway. (C) represents the interactions of ssc-miR-133a-3p, ssc-miR-1 and ssc-miR-486 with the transcription factors binding to them. The yellow nodes indicate the transcription factors, and the pink nodes indicate the miRNAs. 
miRNAs and their target genes that were significantly enriched in the GO and KEGG analyses and the target genes that were reported to be related to lipid metabolism in previous studies.

\section{Transcription factor binding sites (TFBS) prediction of the differentially expressed miRNAs}

The cis-acting elements that regulate gene expression are distributed in the 300-3000 bp upstream of the coding region. Taking into consideration of the methods described by Liu et al. [67], 2000 bp upstream of ssc-miR-486, ssc-miR-133a-3p and ssc-miR-1 were selected as the promoter sequences and were downloaded from Ensembl (http://uswest.ensembl.org/) and NCBI Database (http:/www.ncbi.nlm.nih.gov/). Then, these sequences were submitted to JASPAR CORE Vertebrate (http://jaspardev.genereg.net) and Scan Version 1.7 (http:// www-bimas.cit.nih.gov/molbio/proscan/) for in silico analysis.

\section{qRT-PCR identification}

The qRT-PCR method was utilized to validate the expression of the miRNAs. For each miRNA, three biological replications were performed. Briefly, $2 \mu \mathrm{g}$ of total RNA was reverse transcribed to cDNA using SG One-Step miRNA RT Kit (\#Q1014, SinoGene, China), and then, SYBR Green real-time PCR was carried out using the synthesized cDNA as the template. The qRTPCR reaction system comprised of $7.5 \mu 1$ of $2 \times$ SG Green qRT-PCR Mix, $0.25 \mu$ l Forward Primer, $0.25 \mu$ l of Reverse Primer, $1 \mu \mathrm{l}$ cDNA and $6 \mu 1$ nuclease-free Water, and the qRT-PCR reactions were performed by Step One PLUS (Applied Biosystems, Foster city, CA), with a condition of $95^{\circ} \mathrm{C}$ for $10 \mathrm{~min}$, followed by 45 cycles of $95^{\circ} \mathrm{C}$ for $15 \mathrm{~s}$ and $60^{\circ} \mathrm{C}$ for $15 \mathrm{~s}$, and a final stage of dissociation analysis. The porcine U6 gene was used as an internal control, and the $\Delta \Delta \mathrm{Ct}$ method was used to calculate the relative expression level of the miRNAs between the samples. The qRT-PCR results produced the amplification plot of an S type and the dissolution curve of a single peak, indicating that specific amplifications were attained with the primers designed by Sinogene Scientific (Beijing, China).

\section{Statistical analyses}

All of the data are presented as the means \pm SD. For the comparisons, a Student's $t$-test was performed, and a corrected $p<0.05$ was considered statistically significant.

\section{CONCLUSIONS}

Taken together, our study explored the potential role of differentially expressed miRNAs in fat deposition in pigs. Thirty-nine known miRNAs and 55 novel miRNAs were identified. The Gene Ontology and KEGG analyses indicated that the target genes of the differentially expressed miRNAs were involved in the PPAR, MAPK and Wnt signaling pathways and were related with lipometabolism. In addition, we first reported that ssc-miR-133a-3p, ssc-miR-486 and sscmiR-1 regulate the target genes CPT1A, SCD5 and SCD, respectively, in the PPAR signaling pathway so that they can have an impact on lipidosis. These three miRNAs were upregulated in the Laiwu pig compared to the Large White pig. Regulation of the target genes CPT1A, SCD5 and SCD by ssc-miR-133a-3p, sscmiR-486 and ssc-miR-1, respectively, may contribute to the molecular mechanism by which Laiwu pigs accumulate more fat when compared to the Large White pigs. Of course, these three targets and the mechanism by which these miRNAs regulate fat deposition still need to be verified in future experiments. Our findings, both computational and experimental, provide valuable information regarding the miRNAs involved in fat deposition and contribute to revealing the molecular mechanism of the gene regulation governing the development and physiology of adipose tissue in pigs. Moreover, our study provides data that might reveal insight into obesity-related diseases.

\section{Author contributions}

X.Y.M. conceived and designed the study and wrote the paper. X.X.Z. performed the experiment and data analysis and wrote the paper; W.L.H. and Y.T.G. performed the experiments and interpreted the data. All the authors read and approved the final manuscript.

\section{CONFLICTS OF INTEREST}

The authors declare that they have no competing interests.

\section{FUNDING}

This work was supported by a grant from the Agricultural Science and Technology Innovation Program (ASTIPIAS05), the National Basic Research and Development Program of China (973 Program) (No. 2015CB943100) and the Basic Research Fund for Central Public Research Institutes of CAAS (No. 2013ywf-zd-2).

\section{REFERENCES}

1. Wu VJ, Pang D, Tang WW, Zhang X, Li L, You Z. Obesity, age, ethnicity, and clinical features of prostate cancer patients. American journal of clinical and experimental urology. 2017; 5:1. 
2. Smitka K, Marešová D. Adipose tissue as an endocrine organ: an update on pro-inflammatory and anti-inflammatory microenvironment. Prague Med Rep. 2015; 116:87-111.

3. Bartel DP. MicroRNAs: genomics, biogenesis, mechanism, and function. cell. 2004; 116:281-97.

4. Vimalraj S, Selvamurugan N. Regulation of proliferation and apoptosis in human osteoblastic cells by microRNA$15 \mathrm{~b}$. International journal of biological macromolecules. 2015; 79:490-7.

5. Zhao M, Sun L, Chen S, Li D, Zhang L, He P, Liu X, Zhang L, Zhang H, Yang D. Borna disease virus infection impacts microRNAs associated with nervous system development, cell differentiation, proliferation and apoptosis in the hippocampi of neonatal rats. Molecular medicine reports. 2015; 12:3697-703.

6. Xu P, Vernooy SY, Guo M, Hay BA. The Drosophila microRNA Mir-14 suppresses cell death and is required for normal fat metabolism. Current Biology. 2003; 13:790-5.

7. Xue Z, Zhao J, Niu L, An G, Guo Y, Ni L. Up-regulation of miR-300 promotes proliferation and invasion of osteosarcoma by targeting BRD7. PloS one. 2015; 10:e0127682.

8. Esau C, Kang X, Peralta E, Hanson E, Marcusson EG, Ravichandran LV, Sun Y, Koo S, Perera RJ, Jain R. MicroRNA-143 regulates adipocyte differentiation. Journal of Biological Chemistry. 2004; 279:52361-5.

9. Liu SY, Zhang YY, Gao Y, Zhang LJ, Chen HY, Zhou Q, Chai ML, Li QY, Jiang H, Yuan B. MiR-378 plays an important role in the differentiation of bovine preadipocytes. Cellular Physiology and Biochemistry. 2015; 36:1552-62.

10. Wang K, Li W, Bai Y, Yang W, Ling Y, Fang M. ssc-miR7134-3p regulates fat accumulation in castrated male pigs by targeting MARK4 gene. International journal of biological sciences. 2017; 13:189.

11. Peng Y, Chen FF, Ge J, Zhu JY, Shi XE, Li X, Yu TY, Chu GY, Yang GS. miR-429 Inhibits Differentiation and Promotes Proliferation in Porcine Preadipocytes. International journal of molecular sciences. 2016; 17:2047.

12. Jokinen MP, Clarkson TB, Prichard RW. Animal models in atherosclerosis research. Experimental and molecular pathology. 1985; 42:1-28.

13. Carey GB. The swine as a model for studying exerciseinduced changes in lipid metabolism. Medicine and science in sports and exercise. 1997; 29:1437-43.

14. Miao X, Luo Q, Qin X, Guo Y, Zhao H. Genome-wide mRNA-seq profiling reveals predominant down-regulation of lipid metabolic processes in adipose tissues of Small Tail Han than Dorset sheep. Biochemical and biophysical research communications. 2015; 467:413-20.

15. Miao X, Luo Q. Genome-wide transcriptome analysis between small-tail Han sheep and the Surabaya fur sheep using highthroughput RNA sequencing. Reproduction. 2013; 145:587-96.

16. Miao X, Luo Q, Qin X. Genome-wide transcriptome analysis in the ovaries of two goats identifies differentially expressed genes related to fecundity. Gene. 2016; 582:69-76.
17. Miao X, Luo Q, Zhao H, Qin X. Genome-wide analysis of miRNAs in the ovaries of Jining Grey and Laiwu Black goats to explore the regulation of fecundity. Scientific reports. 2016; 6 .

18. Miao X, Qin QLX. Genome-wide transcriptome analysis of mRNAs and microRNAs in Dorset and Small Tail Han sheep to explore the regulation of fecundity. Molecular and cellular endocrinology. 2015; 402:32-42.

19. Miao X, Luo Q, Qin X. Genome-wide analysis reveals the differential regulations of mRNAs and miRNAs in Dorset and Small Tail Han sheep muscles. Gene. 2015; 562:188-96.

20. Miao X, Luo Q, Zhao H, Qin X. Ovarian transcriptomic study reveals the differential regulation of miRNAs and lncRNAs related to fecundity in different sheep. Scientific reports. 2016; 6:35299.

21. Miao X, Luo Q, Zhao H, Qin X. Co-expression analysis and identification of fecundity-related long non-coding RNAs in sheep ovaries. Scientific reports. 2016; 6 .

22. Guo Y, Zhang X, Huang W, Miao X. Identification and characterization of differentially expressed miRNAs in subcutaneous adipose between Wagyu and Holstein cattle. Scientific Reports. 2017; 7.

23. Huang W, Guo Y, Du W, Zhang X, Li A, Miao X. Global transcriptome analysis identifies differentially expressed genes related to lipid metabolism in Wagyu and Holstein cattle. Scientific Reports. 2017; 7.

24. Liu L, Qian K, Wang C. Discovery of porcine miRNA 196a/b may influence porcine adipogenesis in longissimus dorsi muscle by miRNA sequencing. Animal genetics. 2017; 48:175-81.

25. Sheng X, Wang L, Ni H, Wang L, Qi X, Xing S, Guo Y. Comparative Analyses between Skeletal Muscle miRNAomes from Large White and Min Pigs Revealed MicroRNAs Associated with Postnatal Muscle Hypertrophy. PloS one. 2016; 11:e0156780.

26. Gaffo E, Zambonelli P, Bisognin A, Bortoluzzi S, Davoli R. miRNome of Italian Large White pig subcutaneous fat tissue: new miRNAs, isomiRs and moRNAs. Animal genetics. 2014; 45:685-98.

27. Madsen L, Petersen RK, Kristiansen K. Regulation of adipocyte differentiation and function by polyunsaturated fatty acids. Biochimica et Biophysica Acta (BBA)Molecular Basis of Disease. 2005; 1740:266-86.

28. Miller WL, Bose HS. Early steps in steroidogenesis: intracellular cholesterol trafficking thematic review series: genetics of human lipid diseases. Journal of lipid research. 2011; 52:2111-35.

29. Gao X, Li K, Hui X, Kong X, Sweeney G, Wang Y, Xu A, Teng M, Liu P, Wu D. Carnitine palmitoyltransferase 1A prevents fatty acid-induced adipocyte dysfunction through suppression of c-Jun N-terminal kinase. Biochemical Journal. 2011; 435:723-32.

30. Ng M, Fleming $\mathrm{T}$, Robinson $\mathrm{M}$, Thomson B, Graetz N, Margono C, Mullany EC, Biryukov S, Abbafati C, Abera 
SF. Global, regional, and national prevalence of overweight and obesity in children and adults during 1980-2013:a systematic analysis for the Global Burden of Disease Study 2013. The lancet. 2014; 384:766-81.

31. Tchernof A, Després JP. Pathophysiology of human visceral obesity: an update. Physiological reviews. 2013; 93:359 404.

32. Kim B, Choi KM, Yim HS, Lee MG. Ascorbic acid enhances adipogenesis of 3T3-L1 murine preadipocyte through differential expression of collagens. Lipids in health and disease. 2013; 12:182.

33. Jeong YS, Hong JH, Cho KH, Jung HK. Grape skin extract reduces adipogenesis-and lipogenesis-related gene expression in 3T3-L1 adipocytes through the peroxisome proliferator-activated receptor- $\gamma$ signaling pathway. Nutrition research. 2012; 32:514-21.

34. Peterson JM, Seldin MM, Tan SY, Wong GW. CTRP2 overexpression improves insulin and lipid tolerance in dietinduced obese mice. PLoS One. 2014; 9:e88535.

35. Li H, Chen X, Guan L, Qi Q, Shu G, Jiang Q, Yuan L, Xi Q, Zhang Y. MiRNA-181a regulates adipogenesis by targeting tumor necrosis factor- $\alpha$ (TNF- $\alpha)$ in the porcine model. PloS one. 2013; 8:e71568.

36. Kim YJ, Hwang SJ, Bae YC, Jung JS. MiR - 21 Regulates Adipogenic Differentiation through the Modulation of TGF - $\beta$ Signaling in Mesenchymal Stem Cells Derived from Human Adipose Tissue. Stem cells. 2009; 27:3093-102.

37. Karbiener M, Fischer C, Nowitsch S, Opriessnig P, Papak C, Ailhaud G, Dani C, Amri EZ, Scheideler M. microRNA miR-27b impairs human adipocyte differentiation and targets PPAR $\gamma$. Biochemical and biophysical research communications. 2009; 390:247-51.

38. Sun T, Fu M, Bookout AL, Kliewer SA, Mangelsdorf DJ. MicroRNA let-7 regulates 3T3-L1 adipogenesis. Molecular endocrinology. 2009; 23:925-31.

39. Luo Y, Wu X, Ling Z, Yuan L, Cheng Y, Chen J, Xiang C. microRNA133a targets Foxl2 and promotes differentiation of $\mathrm{C} 2 \mathrm{C} 12$ into myogenic progenitor cells. DNA and cell biology. 2015; 34:29-36.

40. Townley-Tilson WD, Callis TE, Wang D. MicroRNAs 1, 133, and 206: critical factors of skeletal and cardiac muscle development, function, and disease. The international journal of biochemistry \& cell biology. 2010; 42:1252-5.

41. Lin JC. RBM4-MEF2C network constitutes a feedforward circuit that facilitates the differentiation of brown adipocytes. RNA Biol. 2015; 12:208-20.

42. Liu W, Bi P, Shan T, Yang X, Yin H, Wang YX, Liu N, Rudnicki MA, Kuang S. miR-133a regulates adipocyte browning in vivo. PLoS genetics. 2013; 9:e1003626.

43. Prats-Puig A, Ortega FJ, Mercader JM, Moreno-Navarrete JM, Moreno M, Bonet N, Ricart W, López-Bermejo A, Fernández-Real JM. Changes in circulating microRNAs are associated with childhood obesity. The Journal of Clinical Endocrinology \& Metabolism. 2013; 98:E1655-E60.
44. Moore KJ, Rayner KJ, Suárez Y, Fernández-Hernando C. The role of microRNAs in cholesterol efflux and hepatic lipid metabolism. Annual review of nutrition. 2011; 31:49-63.

45. Rotllan N, Fernández-Hernando C. MicroRNA regulation of cholesterol metabolism. Cholesterol. 2012; 2012.

46. Ramírez CM, Rotllan N, Vlassov AV, Dávalos A, Li M, Goedeke L, Aranda JF, Cirera-Salinas D, Araldi E, Salerno A. Control of cholesterol metabolism and plasma HDL levels by miRNA-144. Circulation research. 2013:CIRCRESAHA. 112.300626.

47. Huang J, Zhao L, Xing L, Chen D. MicroRNA - 204 regulates Runx2 protein expression and mesenchymal progenitor cell differentiation. Stem cells. 2010; 28:357-64.

48. Zheng Z, Yan D, Chen X, Huang H, Chen K, Li G, Zhou L, Zheng D, Tu L, Da Dong X. MicroRNA-206: effective Inhibition of gastric cancer progression through the c-Met pathway. PloS one. 2015; 10:e0128751.

49. Epis MR, Giles KM, Barker A, Kendrick TS, Leedman PJ. miR-331-3p regulates ERBB-2 expression and androgen receptor signaling in prostate cancer. Journal of Biological Chemistry. 2009; 284:24696-704.

50. Nishikawa R, Goto Y, Sakamoto S, Chiyomaru T, Enokida H, Kojima S, Kinoshita T, Yamamoto N, Nakagawa M, Naya Y. Tumor - suppressive microRNA - 218 inhibits cancer cell migration and invasion via targeting of LASP1 in prostate cancer. Cancer science. 2014; 105:802-11.

51. Zhao Z, Qin L, Li S. miR-411 contributes the cell proliferation of lung cancer by targeting FOXO1. Tumor Biology. 2016; 37:5551-60.

52. Gwon SY, Ahn JY, Jung CH, Moon BK, Ha TY. Shikonin suppresses ERK 1/2 phosphorylation during the early stages of adipocyte differentiation in 3T3-L1 cells. BMC complementary and alternative medicine. 2013; 13:207.

53. Aouadi M, Jager J, Laurent K, Gonzalez T, Cormont M, Binétruy B, Marchand-Brustel L, Tanti JF, Bost F. p38MAP Kinase activity is required for human primary adipocyte differentiation. FEBS letters. 2007; 581:5591-6.

54. Tominaga S, Yamaguchi T, Takahashi SI, Hirose F, Osumi T. Negative regulation of adipogenesis from human mesenchymal stem cells by Jun N-terminal kinase. Biochemical and biophysical research communications. 2005; 326:499-504.

55. Zhu H, Guariglia S, Li W, Brancho D, Wang ZV, Scherer $\mathrm{PE}$, Chow CW. Role of extracellular signal-regulated kinase 5 in adipocyte signaling. Journal of Biological Chemistry. 2014; 289:6311-22.

56. Bowers RR, Lane MD. Wnt signaling and adipocyte lineage commitment. Cell Cycle. 2008; 7:1191-6.

57. Prestwich TC, MacDougald OA. Wnt/ $\beta$-catenin signaling in adipogenesis and metabolism. Current opinion in cell biology. 2007; 19:612-7.

58. Kennell JA, MacDougald OA. Wnt signaling inhibits adipogenesis through $\beta$-catenin-dependent and-independent mechanisms. Journal of Biological Chemistry. 2005; 280:24004-10. 
59. Wu X, Zou X, Chang Q, Zhang Y, Li Y, Zhang L, Huang J, Liang B. The evolutionary pattern and the regulation of stearoyl-CoA desaturase genes. BioMed research international. 2013; 2013.

60. Sampath H, Miyazaki M, Dobrzyn A, Ntambi JM. StearoylCoA desaturase-1 mediates the pro-lipogenic effects of dietary saturated fat. Journal of Biological Chemistry. 2007; 282:2483-93.

61. Rincon G, Islas-Trejo A, Castillo AR, Bauman DE, German BJ, Medrano JF. Polymorphisms in genes in the SREBP1 signalling pathway and SCD are associated with milk fatty acid composition in Holstein cattle. Journal of Dairy Research. 2012; 79:66-75.

62. Malandrino MI, Fucho R, Weber M, Calderon-Dominguez M, Mir JF, Valcarcel L, Escoté X, Gómez-Serrano M, Peral B, Salvadó L. Enhanced fatty acid oxidation in adipocytes and macrophages reduces lipid-induced triglyceride accumulation and inflammation. American Journal of Physiology-Endocrinology and Metabolism. 2015; 308:E756-E69.
63. Souza-Mello V. Peroxisome proliferator-activated receptors as targets to treat non-alcoholic fatty liver disease. World journal of hepatology. 2015; 7:1012.

64. Veiga FMS, Graus-Nunes F, Rachid TL, Barreto AB, Mandarim-de-Lacerda CA, Souza-Mello V. Antiobesogenic effects of WY14643 (PPAR-alpha agonist): Hepatic mitochondrial enhancement and suppressed lipogenic pathway in diet-induced obese mice. Biochimie. 2017; 140:106-16.

65. Wang Z, Gerstein M, Snyder M. RNA-Seq: a revolutionary tool for transcriptomics. Nature reviews genetics. 2009; 10:57-63.

66. Ambros V, Bartel B, Bartel DP, Burge CB, Carrington JC, Chen X, Dreyfuss G, Eddy SR, Griffiths-Jones S, Marshall M. A uniform system for microRNA annotation. Rna. 2003; 9:277-9.

67. Liu Q, Wang H, Zhang Z, Wu J, Feng Y, Zhu Z. Divergence in function and expression of the NOD26-like intrinsic proteins in plants. BMC genomics. 2009; 10:313. 